\title{
Excitability of Spinal Neural Function by Motor Imagery with Isometric Opponens Pollicis Activity: Influence of Difference Methods of Motor Imagery
}

Marina Todo ${ }^{1 *}$, Yoshibumi Bunnno ${ }^{1,2}$ and Toshiaki Suzuki ${ }^{1,2}$

${ }^{1}$ Clinical Physical Therapy Laboratory, Faculty of Health Sciences, Kansai University of Health Sciences, Osaka, Japan

${ }^{2}$ Graduate School of Kansai University of Health Sciences, 2-11-1 Wakaba, Sennangun Kumatori, Osaka 590-0433, Japan

\section{Abstract}

Purpose: This study aimed to examine the effect on spinal nerve function that differences in the motor image method.

Methods: We recorded the F-wave during three different methods of MI from previous research as follows. Vision imagery is the method that subjects image the digital digits to display pinch force. Muscle imagery is the method that subjects image the muscle contraction during pinching. Sensory imagery is the method that subjects image the feeling you hold down the pinch sensor.

Results: Both persistence and F/M amplitude ratios motor imagery under vision-image, muscle-image and sensory-image significantly higher than that observed at rest. No significant were noted in relative data for amplitude ratio of $\mathrm{F} / \mathrm{M}$ and persistence between motor imagery that three image way.

Conclusion: Motor imagery under three different methods as vision-image or muscle-image and sensory-image was no significant difference in the excitability of spinal neural function between the three MI methods condition. However excitability of spinal neural function during imaging under all conditions tended to be higher during motor imager than at rest.

Keywords: Motor imagery; F-wave; Motor imagery method

\section{Introduction}

Motor imagery, the mental rehearsal of motor acts without overt movement, has been shown to improve motor performance in healthy subjects [1] and benefit motor function recovery following stroke [2,3]. This line stroke with Ryding et al. [2], mention the effectiveness of activation of cerebellum during motor imagery against healthy person. Stevens et al. [3], has expressed effectiveness of motor imagery that middle cerebral artery stroke that resulted in chronic hemiparesis. The effects of motor imagery have been discussed in many neurophysiological studies using motor-evoked potentials (MEPs). Brain activity due to the motor image (MI) has been reported several studies using such as functional magnetic resonance imaging (fMRI) and transcranial magnetic stimulation (TMS). Local brain activation when actually moving is reported to be activated as well by MI [4]. Results of research on the excitability of spinal neural function during MI has not been fixed. Hale et al. [5], were measured H-wave of soleus muscle during MI. Results show that first H-wave during MI did not change but by repeating the MI, the amplitude of the $\mathrm{H}$ wave is increased [5]. Suzuki et al. [6], were monitored F-wave of opponens pollicis muscles during MI when subjects were instructed to hold the sensor between the thumb and index finger. And mimic the number of contractions displayed by the monitor while looking at the display with their eyes either open or close [6]. Results show that F-wave during $\mathrm{MI}$ is increased. However, the excitability of the cerebral cortex during MI may result from an increase in the MEP amplitude as measured by TMS; spinal neural function has been reported not excited [7].

When MI is used as part of neurological rehabilitation, it has the potential to increase both motor cortical and spinal neural function. In turn, improved spinal neural function can result in improved muscle function. Our ultimate goal of this MI research was to find the best method of improving the excitability of spinal neural function in the clinical setting. In other words, setting the condition of MI can be considered to affect the excitability of spinal nerve function. In addition, there is a possibility that difference of methods of motor imagery affects the excitability of spinal neural function. In recently, it is said that the effect of MI is difference in each subjects. We think that the reason is difference of method in MI. Therefore, we interviewed the contents that motor image from subjects. The result could be classified into three types (vision image, muscle image, and sensory image). In this study, we give one task from three types and get motor image so we monitored the F-wave.

\section{Materials and Methods}

\section{Subjects}

A total of 20 healthy volunteers ( 8 males and 12 females; mean age, 21.5 years) participated in the study. This study was approved the Research Ethics Committee at Kansai University of Health Sciences. The experiments were conducted in accordance with the Declaration of Helsinki. This study was not concerned in Conflict of Interests.

\section{F-wave during motor imagery}

Subjects were comfortably maintained in a supine position with both shoulders rotated. After stimulating median nerve at the wrist the non-dominant hand at rest and under motor imagery conditions, a Viking Quest electromyography machine (NATUS, USA) was used to record the F-waves from the thenar muscles in the non-dominant

*Corresponding author: Marina Todo, Clinical Physical Therapy Laboratory, Faculty of Health Sciences, Kansai University of Health Sciences, Osaka, Japan, Tel: 81724538374; Fax: 81724538798; E-mail: todo@kansai.ac.jp

Received November 17, 2016; Accepted December 05, 2016; Published December 12, 2016

Citation: Todo M, Bunnno Y, Suzuki T (2016) Excitability of Spinal Neural Function by Motor Imagery with Isometric Opponens Pollicis Activity: Influence of Difference Methods of Motor Imagery. Int J Neurorehabilitation 3: 232. doi: 10.4172/23760281.1000232

Copyright: $\odot 2016$ Todo M, et al. This is an open-access article distributed under the terms of the Creative Commons Attribution License, which permits unrestricted use, distribution, and reproduction in any medium, provided the original author and source are credited. 
Citation: Todo M, Bunnno Y, Suzuki T (2016) Excitability of Spinal Neural Function by Motor Imagery with Isometric Opponens Pollicis Activity: Influence of Difference Methods of Motor Imagery. Int J Neurorehabilitation 3: 232. doi: 10.4172/2376-0281.1000232

Page 2 of 4

side with a pair of round disks attached to the skin, with collodion over the abdomen of the thenar muscle and the metacarpophalangeal joint of the thumb. The maximal stimulus was determined by delivering $0.2 \mathrm{~ms}$ square-wave pulses of increasing intensity to elicit the largest compound muscle action potentials. Supramaximal shocks (adjusted up to the value of $20 \%$ higher than the maximal stimulus) were delivered at $0.5 \mathrm{~Hz}$ for acquisition of F-waves. As a treatment before you put on skin the electrode, the skin was prepared with abrasive gel to maintain the impedance below $5 \mathrm{~K} \Omega$. The stimulating electrodes comprised a cathode placed over the left median nerve at $3 \mathrm{~cm}$ proximal to the palmar crease of the wrist joint and an anode placed $2 \mathrm{~cm}$ further proximally. All subjects underwent a training procedure prior to the actual experiment. First, subjects, while looking the numbers pinch force value is displayed, learned $50 \% \mathrm{MVC}$ of confrontation movement by thumb and index finger. After taking sufficient rest, we recorded the F-wave during three different methods of MI from previous research as follows. Vison imagery is the method that subjects image the digital digits to display pinch force. Muscle imagery is the method that subjects image the muscle contraction during pinching. Sensory imagery is the method that subjects image the feeling you hold down the pinch sensor.

\section{Data analysis}

The F-wave was analyzed with respect to persistence, amplitude ratio of $\mathrm{F} / \mathrm{M}$, and latency. Persistence was defined as the number of measurable $\mathrm{F}$-wave responses divided by 30 trials of supramaximal stimulation. The amplitude ratio of $\mathrm{F} / \mathrm{M}$ was defined as the mean amplitude of all F-waves divided by $\mathrm{M}$-wave amplitude. Latency was defined as the mean time from stimulation to the onset of measurable F-wave. Bonferroni tests were used to compare results between resting condition and each motor imagery conditions under vision-image, muscle-image and sensory-image conditions. A $p$ value of $<0.05$ was considered statistically significant. Wilcoxon tests were used to compare results between resting condition and each image that visionimage, muscle-image and sensory-image by using the relative value was 1 resting.

\section{Results}

Amplitude ratio of F/M during motor imagery under visionimage, muscle-image and sensory-image tended to increase compared with that observed at rest (Bonfferioni tests; $\mathrm{p}<0.01$ ) (Table 1 and Figures 1 and 2). The persistence during motor imagery that visionimage, muscle-image and sensory-image significantly higher than that observed at rest (Bonfferioni tests; $\mathrm{p}<0.01$ ) (Table 1 and Figures 1 and $3)$. No significant differences were noted in latency in the three motor imagery conditions (Table 1 and Figures 1 and 4).

Relative data for persistence during motor imagery under visionimage, muscle-image and sensory-image were not significant (Table 2 and Figure 5). No significant were noted in relative data for amplitude ratio of $\mathrm{F} / \mathrm{M}$ and latency between motor imagery that three image way (Table 2 and Figures 6 and 7).

\section{Discussion}

There are many reports that the excitability of the cerebral cortex obtained during MI $[3,4]$. Almost reports that excitability of cortical function is increasing. However, excitability of spinal neural function during MI has been a variety of reports from previous studies. Suzuki

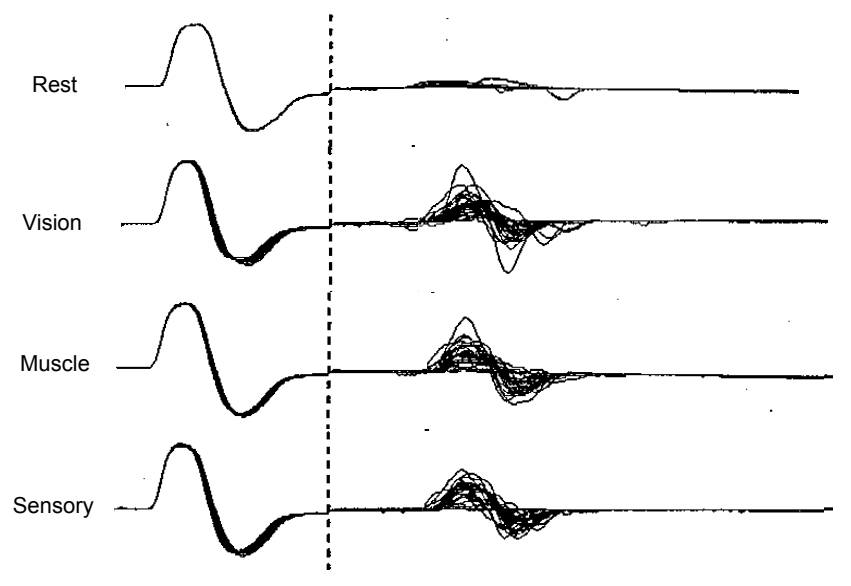

Figure 1: Specific F-wave during motor imagery.

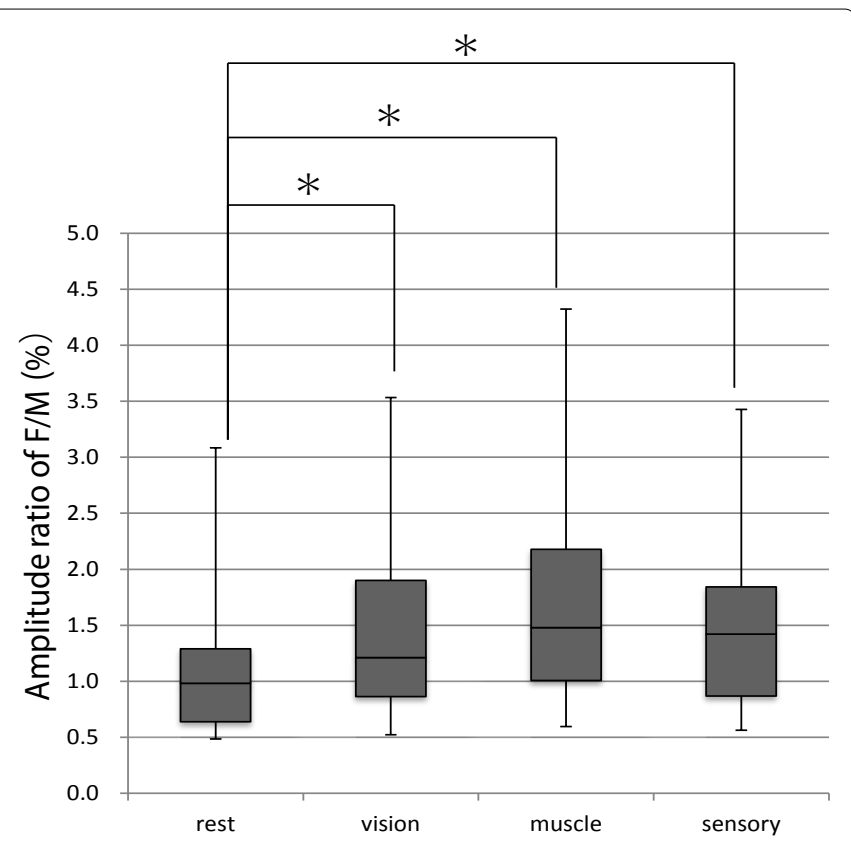

Figure 2: Difference of methods of motor image F-wave.

Amplitude ratio of $F / M$ during rest and motor imagery with vision-image or muscle image and sensory-image. Amplitude ratio of F/M during imaging under all conditions tended to be higher during motor imager than at rest $\left({ }^{*} \mathrm{p}<0.01\right)$

Table 1: Difference of methods of motor image F-wave.

\begin{tabular}{|l|c|c|c|}
\hline & Rest & Vision & Muscle \\
\hline Persistence (\%) & $64.63( \pm 21.85)$ & $73.78( \pm 19.10)^{*}$ & $77.88( \pm 18.05)^{*}$ \\
\hline F/M amplitude ratio (\%) & $0.96( \pm 0.58)$ & $1.36( \pm 0.85)^{*}$ & $1.52( \pm 1.00)^{*}$ \\
\hline Latency (ms) & $25.01( \pm 4.86)$ & $24.07( \pm 1.88)$ & $1.31( \pm 0.75)^{*}$ \\
\hline
\end{tabular}

Mean \pm SD. ${ }^{*} p<0.01,{ }^{* *} p<0.05$ vs. at rest

Persistence and amplitude ratio of F/M during the three motor imagery methods were significantly higher than those at rest. Latency was not significantly different among all trials 
Citation: Todo M, Bunnno Y, Suzuki T (2016) Excitability of Spinal Neural Function by Motor Imagery with Isometric Opponens Pollicis Activity: Influence of Difference Methods of Motor Imagery. Int J Neurorehabilitation 3: 232. doi: 10.4172/2376-0281.1000232

Page 3 of 4

Table 2: Comparison between relative values under the three methods motor imagery.

\begin{tabular}{|l|c|c|c|}
\hline & Vision & Muscle & Sensory \\
\hline Relative data for persistence & $1.19( \pm 0.25)$ & $1.30( \pm 0.37)$ & $1.19( \pm 0.23)$ \\
\hline Relative data for F/M amplitude ratio & $1.46( \pm 0.73)$ & $1.57( \pm 0.62)$ & $1.40( \pm 0.53)$ \\
\hline Relative data for Latency & $0.98( \pm 0.10)$ & $0.98( \pm 0.10)$ & $0.99( \pm 0.10)$ \\
\hline
\end{tabular}

Mean \pm SD

Relative values were not significantly different among the three MI conditions

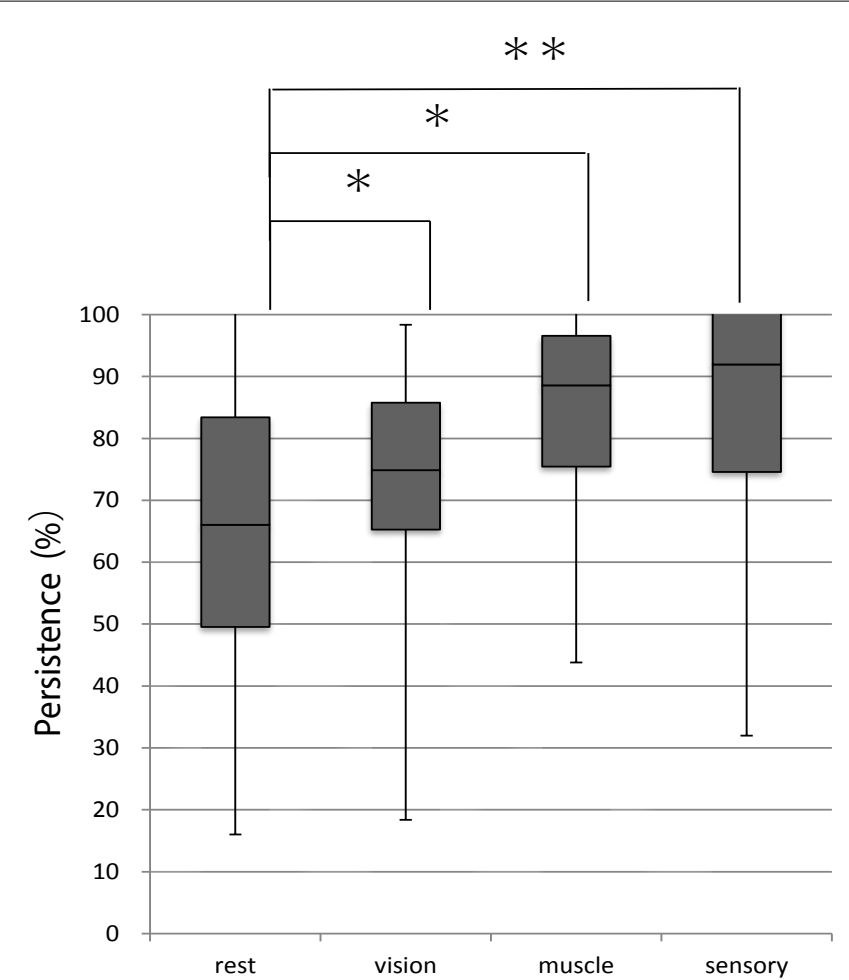

Figure 3: Difference of methods of motor image F-wave.

Persistence during rest and motor imagery with vision-image or muscle- image and sensory image. Persistence during imaging under all conditions tended to be higher during motor imager than at rest $\left({ }^{*} p<0.01{ }^{* *} p<0.05\right)$

et al. was reported that the motor imagery condition using isometrically $50 \%$ MVC of the opponens pollicis muscle by holding sensor of the pinch meter between the thumb and index finger excited spinal neural function [8]. This result shows that MI with preparation of movement is important of condition close to the actual movement. This means that there is a possibility that the typical method of MI can affect the excitability of spinal neural function. We also would like to find the effective method of MI both excitation of spinal and cerebral neuron function. In our previously study, we examined the method of MI due to subjective content using interview. MI method is the same as the research methods of Suzuki et al. [6]. Results show the three types as follows. The first type is vision-image that digital number representing the pinch force. The next type is muscle-image that to image contraction of opponens pollicis muscles when hold the pinch meter. The last type is sensory-image that to image sensory of finger pad when hold the pinch meter.

We examined F-wave, index of the excitability of spinal neural function, during three different methods of MI under vision image, muscle image and sensory image. Amplitude ratio of $\mathrm{F} / \mathrm{M}$ and persistence during MI under vision-image, muscle-image and sensory- image significant increase compared with that observed at rest. However no significant difference among three different methods was noted in relative data for amplitude ratio of $\mathrm{F} / \mathrm{M}$ and persistence.

We hypothesis imagery of the motor output of opponens pollicis muscle and sense of finger as muscle-image and sensory-image more

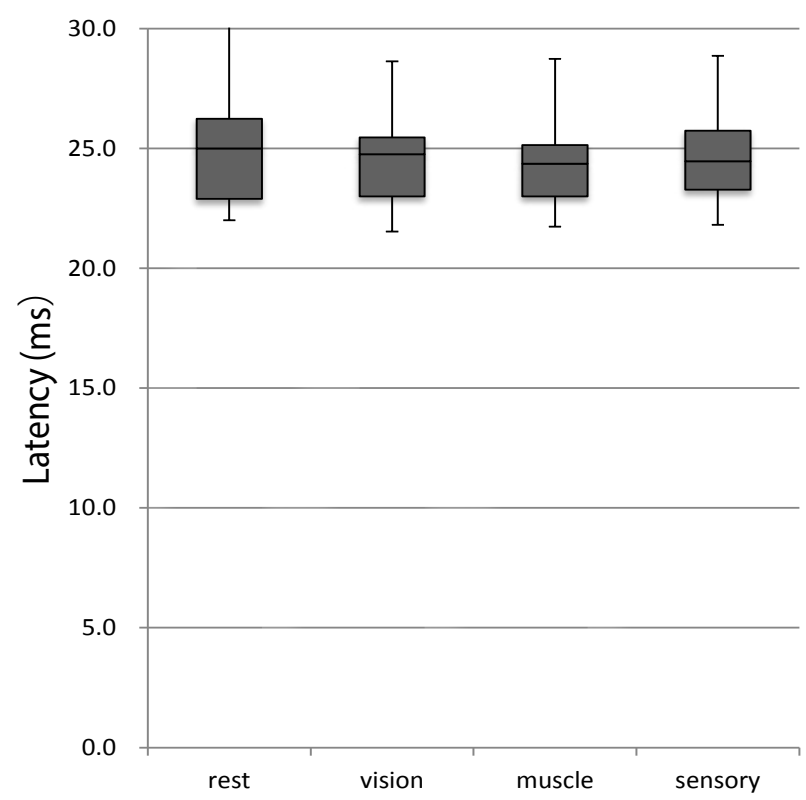

Figure 4: Difference of methods of motor image F-wave.

F-wave latency during rest and motor imagery with vision-image or muscleimage and sensory-image. No significant difference was observed between resting and motor imagery conditions

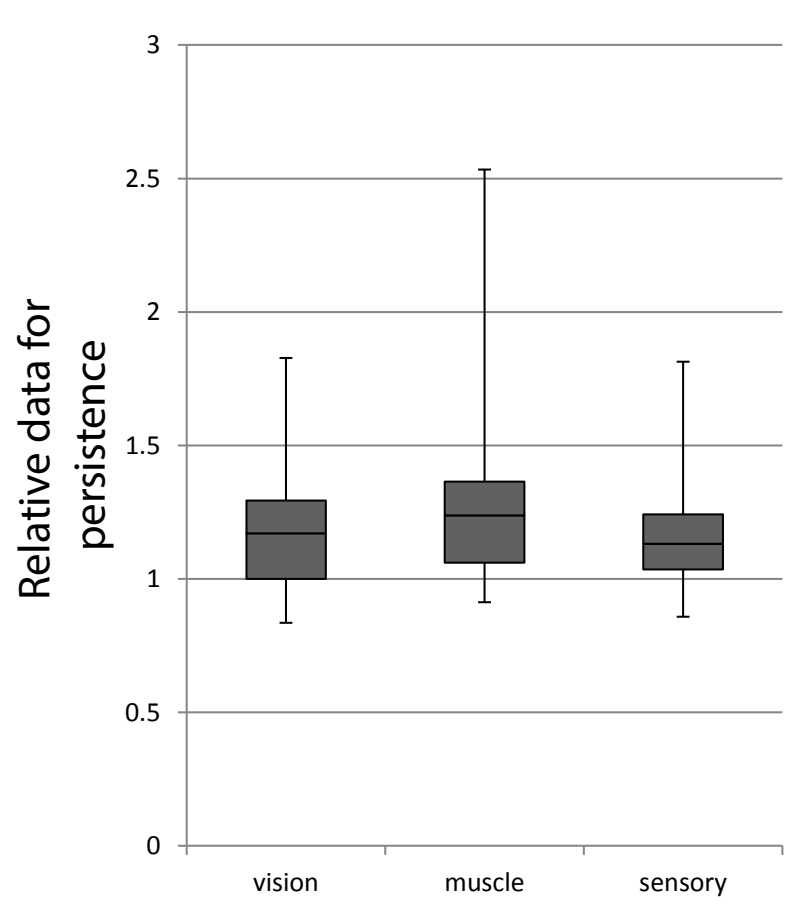

Figure 5: Comparison between relative values under the three methods moto imagery. 
Citation: Todo M, Bunnno Y, Suzuki T (2016) Excitability of Spinal Neural Function by Motor Imagery with Isometric Opponens Pollicis Activity: Influence of Difference Methods of Motor Imagery. Int J Neurorehabilitation 3: 232. doi: 10.4172/2376-0281.1000232

Page 4 of 4

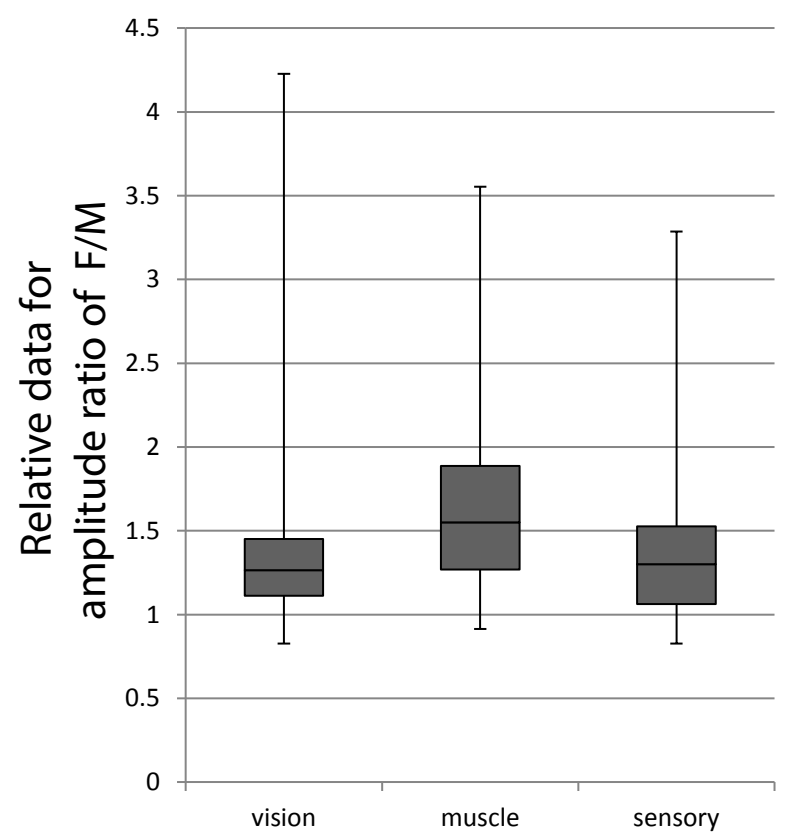

Figure 6: Comparison between relative values under the three methods motor imagery.

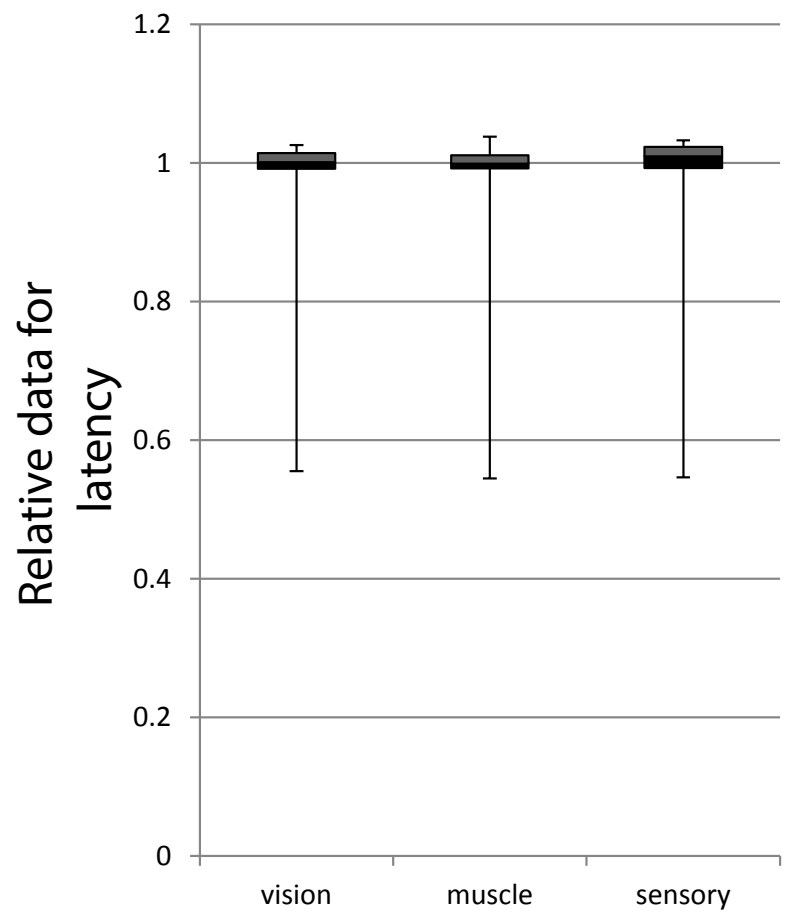

Figure 7: Comparison between relative values under the three methods motor imagery.

facilitated the spinal neuron function rather than that in imagery of the result number of pinch meter obtained from pinch sensor. In addition we had predicted that muscle image and sensory image that is the motor output sites than the vision image is increased of excitability of the spinal nerve function. However, there was no significant difference in three methods MI from this study. In other words, even if the image of the motor output site of opponens pollicis muscle and sense of finger, it is suggested that MI with only result number of pinch meter may excite excitability of spinal nerve function. This reason is the method of motor learning as $50 \%$ MVC confrontation movement by thumb and index finger in this study was adapted the number of pinch force value in displayed. Therefore when the image of only result number of pinch meter, there is possibility participation that causal relationships of motor learning.

\section{Conclusion}

We tested the F-wave from the thenar muscles of the non-dominant side during three different methods as vision-image or muscle-image and sensory-image in healthy subjects. There was no significant difference in the excitability of spinal neural function between the three MI methods condition. However excitability of spinal neural function during imaging under all conditions tended to be higher during motor imager than at rest. Excitability of spinal cord nerve function is increased while trying to image of motor output site, but try to image in clinical scene. In addition, the results were compared with the interviews with previous studies, it is not possible to conclude from the correlation are matched of excitability of spinal cord nerve function and subjective evaluation. In the future, it is necessary to consider the correlation of the excitability of spinal cord nerve function and subjective evaluation, add to task of motor imagery based on the interview results. And it is necessary to research the more efficient motor imagery methods.

\section{References}

1. Pascual-Leone A, Nguyet D, Cohen LG, Brasil-Neto JP, Cammarota A, et al. (1995) Modulation of muscle responses evoked by transcranial magnetic stimulation during the acquisition of new fine motor skills. Journal of Neurophysiology 74: 1037-1045.

2. Ryding E, Decety J, Sjöholm H, Stenberg G, Ingvar DH (1993) Motor imagery activates the cerebellum regionally. A SPECT rCBF study with $99 \mathrm{mTc}-\mathrm{HMPAO}$ Brain research Cognitive Brain Research 1: 94-99.

3. Stevens JA, Stoykov M E (2003) Using motor imagery in the rehabilitation of hemiparesis. Archives of physical Medicine and Rehabilitation 84: 1090-1092.

4. Olivetti Belardinelli M, Palmiero M, Sestieri C, Nardo D, Di Matteo R, et al. (2009) An fMRI investigation on image generation in different sensory modalities: The influence of vividness. Act psychological 132: 190-200.

5. Hale BS, Raglin JS, Koceja DM (2003) Effect of mental imagery of a motor task on the Hoffmann reflex. Behav Brain Res 142: 81-87.

6. Suzuki T, Bunno Y, Onigata C, Tani M, Uragami S (2014) Excitability of spinal neural function by motor imagery with isometric opponens pollicis activity: Influence of vision during motor imagery. NeuroRehabilitation 34: 725-729.

7. Kasai T, Kawai S, Kawanishi M, Yahagi S (1997) Evidence for facilitation of motor evoked potentials (MEPs) induced by motor imagery. Brain Res 744 : 147-150.

8. Suzuki T, Bunno Y, Onigata C, Tani M, Uragami S (2013) Excitability of spinal neural function during several motor imagery tasks involving isometric opponens pollicis activity. NeuroRehabilitation 33: 171-176. 